\title{
Tycho Photometry: Calibration and First Results
}

\author{
V. Großmann \\ Astronomisches Institut der Universität Tübingen, Germany
}

\begin{abstract}
The star mappers on board the ESA Hipparcos satellite will be used as one part of the Tycho project to obtain photometric data of a quasi $B$ and $V$ band magnitude for the 500,000 brightest stars and one broad-band magnitude $T$ for a further 500,000 stars. Approximately 150 million single observations of these stars will be collected during a 4.5-year mission of the satellite.

The accuracy of the photometric data gained is expected to be $0.03 \mathrm{mag}$ on the average at $B=10.5 \mathrm{mag}$ for non-variable stars. We shall get $T$ magnitudes for stars down to a limiting magnitude of about $B=12$ mag depending on the galactic latitude. We will give an overview of the calibration procedure and present some of the first results.
\end{abstract}

\section{Introduction}

The star mapper data from the ESA Hipparcos satellite provide a unique possibility to compile a large photometric catalogue covering both the southern and northern hemisphere using measurements from one single instrument.

The Hipparcos mission, especially the geometry of the star mapper grid, and the processing of the raw photon counts have been described in detail in one recent A\&A issue entirely dedicated to the satellite (Astron. Astrophys. 258, 1992). The Tycho experiment is described in detail in $\mathrm{H} \varnothing \mathrm{g}$ et al. (1992) and references therein. Thus, we will summarize only the points important for photometry and then present an overview on the calibration procedure and some results.

The star mapper experiment determines the Tycho $B_{T}$ and the $V_{T}$ magnitudes for each star which is detectable by the photomultipliers. The $B_{T}$ and $V_{T}$ bands with effective wavelengths of $\lambda=428 \mathrm{~nm}$ and $\lambda=534 \mathrm{~nm}$ are not equivalent to the Johnson $B$ and $V$ bands: they have roughly the same limits but differ in shape (Scales et al. 1992). A broad-band $T$ magnitude covering both the $B_{T}$ and $V_{T}$ bands is also determined. $T$ is defined such that $T=V_{T}$ for $B_{T}-V_{T}=0$. One disadvantage of the experiment is that the integration time for each star crossing the star mapper grid is the same for both bright and faint stars due to the constant scanning velocity of $168.75 \mathrm{arcsec} / \mathrm{s}$. 


\section{The input data to photometry}

Because the star mapper grid consists of four inclined and four vertical slits, (each $0.914^{\prime \prime}$ by $2400^{\prime \prime}$ ) each star crossing the grid will give eight peaks in the raw count data for the $B_{T}$ and $V_{T}$ band, respectively. These peaks may be caused by a star crossing one of the fields of view. These fields, separated by 58 degrees, are lying in the scanning plane of the satellite and therefore are called preceding and following field of view.

The compressed raw counts received from the satellite are converted to photon count rates for each band. By the use of a non-linear filtering method (cf. Bässgen et al. 1992) the so-called raw transits are produced.

Currently we use only a minor fraction of the raw transits including the transits of the photometric standard stars included in the Tycho input catalogue. The final photometric catalogue of the Tycho measurements however will not be based on the current input catalogue of three million stars but on the revised input catalogue of roughly one million stars derived from the data processing of the first year of the mission (Halbwachs et al. 1992).

In order to calibrate our observations we rely on the catalogue of photometric standard stars compiled from about 267,000 measurements (cf. Grenon et al. 1992) and cross-referenced with the Tycho input catalogue to give 65,058 stars (Egret et al. 1992). Out of this sample 21,559 stars remain with more than two sources or observations at different epochs and some of them are rejected because they are flagged as close pairs or known multiple systems in the Tycho input catalogue. Thus the working sample of photometric standards for calibration discussed below contains 13,436 stars.

\section{Calibration and reduction}

The task preceding the calibration is to identify the raw transits with a particular star. This is done by computing a combined probability for all transits attributed to one star transit both in time and in the standard star magnitude. Stars measured with a high background due to the elliptical orbit of the satellite are rejected. After this procedure the transits are split according to their origin: inclined/vertical slits, following/preceding field of view, upper/lower part of the slit system for the $B_{T}, V_{T}$, and $T$ magnitudes, giving 24 possible combinations.

For each combination six calibration parameters are computed: offset, slit abscissa (both linear and quadratic), colour (both linear and quadratic) and a linear combination of slit abscissa and colour.

The mathematical method is discussed in Scales et al. (1992). After the calibration the parameters are checked for consistency (e.g. sudden parameter changes between two sets of observed transits). The transits are then reduced using the calculated six parameters and each transit is merged into a sorted working catalogue. At the 
moment this catalogue contains about 650,000 single transits.

\section{Some results}

We determined calibration parameters from the sample of photometric standard stars and reduced the transits of about 41,000 stars covering a time interval of about 300 days. In this period we found transits of nearly all photometric standards $(96.7 \%)$.

The calibration parameters were plotted for each of the 24 combinations mentioned above and no significant sudden parameter changes could be detected over the period. However there are differences e.g. between inclined and vertical slit observations which show a different offset in all three bands for the upper part of the slit system.

Figure 1 compares the reduced Tycho observations with the magnitudes from the standard star catalogue for about 4250 single transits for one region of the sky. Because this region is located near a scanning node of the satellite, several transits were observed for each star in less than a year generating the stripes in the three plots. More than $98 \%$ of all transits are within the $3 \sigma$ photon noise errors indicated by the solid curves. The slightly worse $T$ magnitudes are a general phenomenon.

Especially in the $B_{T}$ band, it is obvious that there is a lack of Tycho measurements for stars fainter than the 10th magnitude resulting in a shift of the mean of the distribution towards negative differences in Figure 1. This is due to the detection limit of the instrument: if the faintest detectable magnitude is $B_{T}=11.7 \mathrm{mag}$ it is not possible to get fainter measurements even though they are lying within the photon noise.

In Figure 2 we extracted the reduced observations of a 7 th magnitude star which was observed for about four days. Figure 2a shows each single transit in all three bands for the inclined and the vertical slit groups respectively. In Figure $2 \mathrm{~b}$ the same reduced measurements are averaged for time intervals of about 5.9 hours.

Going back from these plots to a bigger data sample (about 6700 single transits) we find the following mean values and $1 \sigma$ errors for the differences between reduced Tycho magnitudes (single transits, vertical slits) and the ground-based measurements for the $B_{T}$ band :

\begin{tabular}{rrrrc} 
range & mag & transits & mean & $\sigma$ \\
\hline 4.5 & 5.5 & 125 & 0.0014 & 0.0269 \\
5.5 & 6.5 & 513 & -0.0018 & 0.0336 \\
6.5 & 7.5 & 1005 & 0.0027 & 0.0495 \\
7.5 & 8.5 & 1083 & 0.0024 & 0.0821 \\
8.5 & 9.5 & 1130 & 0.0042 & 0.1353 \\
9.5 & 10.5 & 744 & -0.0596 & 0.2313 \\
10.5 & 11.5 & 251 & -0.2610 & 0.3451 \\
11.5 & 12.5 & 19 & -0.6841 & 0.5315 \\
\hline
\end{tabular}

The growing deviation of the mean for faint stars can be seen again in the table as 
discussed above. The $1 \sigma$ errors are consistent with the expected pre-launch errors (e.g.. Grenon 1989).
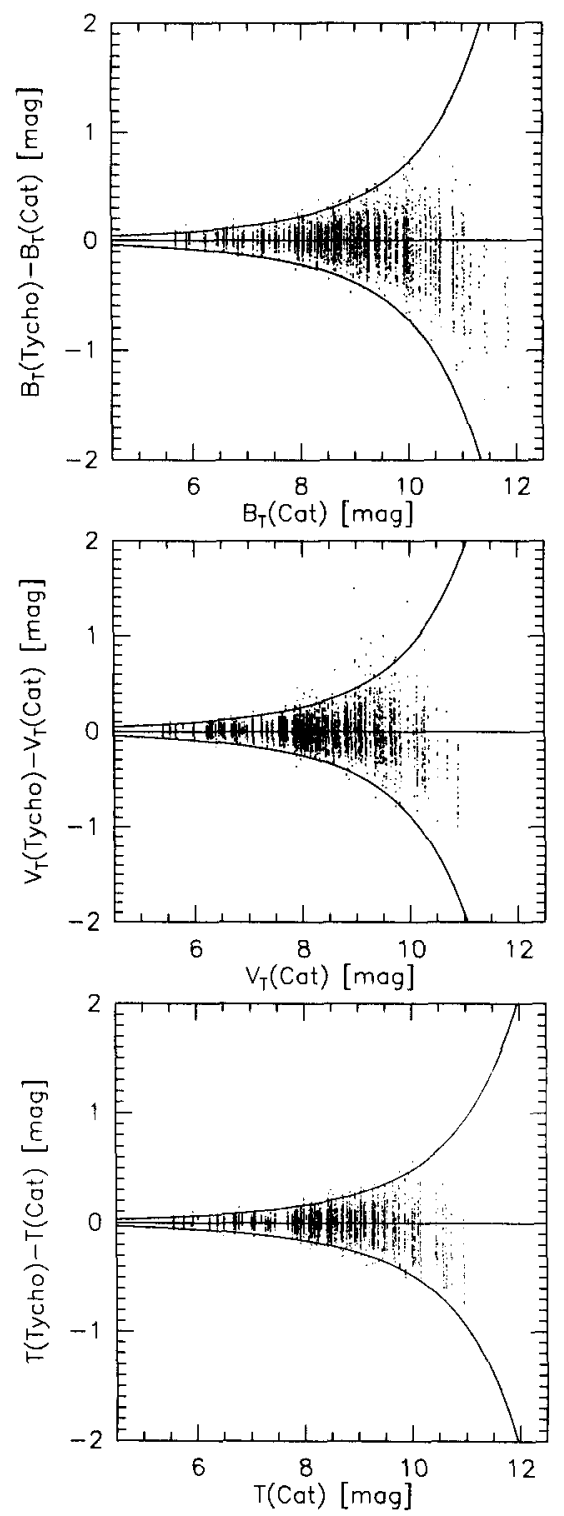

Figure 1 Reduced Tycho observations minus the catalogued values versus the catalogued values for the Tycho $B_{T}, V_{T}$, and $T$ band. The solid curves indicate the $3 \sigma$ limits from photon noise. 

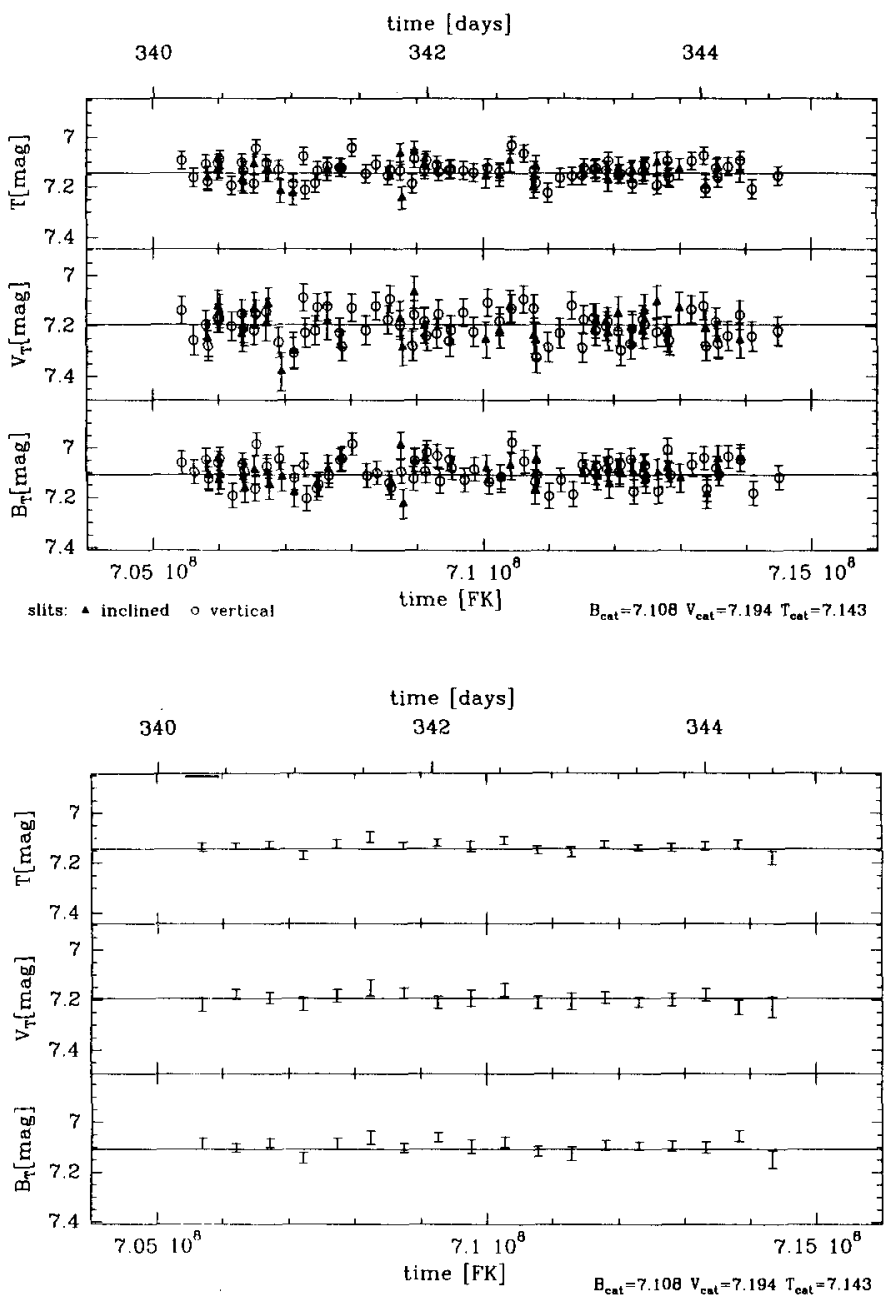

Figure 2 Example of the Tycho measurements for a 7 th magnitude star in all three Tycho bands versus time. a the single transits across the vertical and inclined slit pairs of the Star Mapper grid. b average of these transits for time intervals of 5.9 hours. The lower time axis has filing keys units (one filing key is 0.042 seconds), the upper one gives the corresponding days since the launch of Hipparcos. The solid lines are the magnitudes from the standard star catalogue which are given at the lower right corner of the Figures. The $1 \sigma$ errorbars include the photon noise and the error resulting from the uncertainty in the calibration parameters. 


\section{Current processing and outlook}

On the basis of a time interval of about 300 days we presented some measurements of the star mapper photometry. The on-coming data analysis should yield better results mainly for one reason: in the final data treatment we will use only transits found on the basis of the revised Tycho input catalogue containing roughly one million stars. This will give less false identifications of transits with stars than the usage of the current input catalogue (about three million stars). A minor but important improvement is the better treatment of the background during the detection process.

Due to the decision of ESA this year, the satellite operation will be extended towards mid-1994. As a consequence we will get more (on the average about 150) observations for each star compared with the average 100 observations for a three-year mission.

\section{Acknowledgement}

This work was supported by the Bundesministerium für Forschung und Technologie under grants No. 010085029 .

\section{References:}

Bässgen, G., Wicenec, A., Andreasen, G.K., Høg, E., Wagner, K., Wesselius, P.R., 1991, 258, 186

Egret, D., Didelon, P., McLean, B.J., Russell, J.L., Turon, C., 1992 258, 217

Grenon, M., 1989, ESA SP-1111, Vol. II, 141

Grenon, M., Mermilliod, M., Mermilliod, J.C., 1992, Astron. Astrophys. 258, 88

Halbwachs, J.L., Høg, E., Bastian, U., Hansen, P.C., Schwekendiek, P., Wagner, K., 1992, Astron. Astrophys. 258, 193

Scales, D.R., Snijders, M.A.J., Andreasen, G.K., Grenon,M., Grewing, M. Høg, E., van Leeuwen, F., Lindegren, L., Mauder, H., 1992, Astron. Astrophys. 258, 211

\section{Discussion}

W. Tobin: When do you expect to distribute the optical disks? Also, how many times will an average star be observed in the extended mission

Grossmann: Before the mission was prolonged by ESA we expected to distribute in 1996, now it might be 1997. On average we expect a star will be observed 150 times.

A. T. Young: I am concerned that the standard deviations of the individual measurements are so large. It is very difficult to detect systematic errors smaller than $1 / 3$ or $1 / 4$ of the random errors. I worry that there may be undetected systematic effects larger than a millimagnitude. 